\title{
A Modified Stability Analysis of Two-Dimensional Linear Time Invariant Discrete Systems within the Unity-Shifted Unit Circle
}

\author{
Periyasamy Ramesh \\ Department of Electrical and Electronics Engineering, Anna University, University College of Engineering, \\ Ramanathapuram Campus, Ramanathapuram, India \\ Email: rameshucermd@gmail.com
}

Received 7 March 2016; accepted 22 March 2016; published 25 March 2016

Copyright (C) 2016 by author and Scientific Research Publishing Inc.

This work is licensed under the Creative Commons Attribution International License (CC BY). http://creativecommons.org/licenses/by/4.0/ (c) (i) Open Access

\begin{abstract}
This paper proposes a method to ascertain the stability of two dimensional linear time invariant discrete system within the shifted unit circle which is represented by the form of characteristic equation. Further an equivalent single dimensional characteristic equation is formed from the two dimensional characteristic equation then the stability formulation in the left half of $Z$-plane, where the roots of characteristic equation $f(Z)=0$ should lie within the shifted unit circle. The coefficient of the unit shifted characteristic equation is suitably arranged in the form of matrix and the inner determinants are evaluated using proposed Jury's concept. The proposed stability technique is simple and direct. It reduces the computational cost. An illustrative example shows the applicability of the proposed scheme.
\end{abstract}

\section{Keywords}

Two-Dimensional, Characteristics Equation, Unity Shifting, Inner Determinant, Linear Time Invariant Discrete Systems, Stability

\section{Introduction}

In the last decades a vast amount of research was devoted to the area of two-dimensional systems. The rapid increase of the applicability of the system in area such as image processing, tomography, seismographic data processing, etc. demonstrates the significance of research in this field. In designing a discrete-space system such 
as a digital filter, an important consideration is the stability of the system. From the control view point stability analysis of a 2-D model is also of interest, since a variety of distributed systems, such as time-delay systems, linear multi pass processes and systems governed by certain types of partial differential equations, fit quite naturally in the framework of 2-D system theory. All these initial studies of the stability were carried in frequency domain ( $Z$ domain). More recently, however, the introduction of state-space models for 2-D systems allowed the investigation of the stability in the state-space approach. This is of particular interest due to the recent development of design technique using state-space models. The stability test of 2-D recursive digital filter is mostly numerical computation. The problem of the stable region of filter coefficients has been involved. The stability test is carried out by the new stability test theorem we presented in frequency domain which has the limitation of Jury's and can be used in both conditions of linear and non-linear systems. Further Jury 1971 had presented the positive inner wise and positive definite symmetric matrices for stability of the system. In this method the formulations of symmetric matrices were very complicated and this criterion was rarely used by engineers for high order system. In this present paper a simple and direct scheme is proposed to find stability of linear time invariant discrete systems compared to the Jury (1971) method. The proposed scheme accounts all the coefficient of the unity shifted unit circle one dimensional equivalent characteristic equation in order to form the matrix followed by applying left shifting and right shifting principle to form $\mathrm{X}$ and $\mathrm{Y}$ matrix respectively. The single square matrix $\mathrm{H}=\mathrm{X}+\mathrm{Y}$ has been constructed from $\mathrm{X}$ and $\mathrm{Y}$ matrix with respected to Jury's proposal. For an absolute stable system, the $\mathrm{H}$ value needs to be positive inner-wise which was identified for all its determinants that started from the centre elements and proceeding outwards the need to be positive. Furthermore, one more necessary condition is proposed along with Jury's condition for stability.

\section{Literature Survey}

Recently several contributions have been done to solve the problem in design and analysis of a two dimensional linear time invariant discrete system. Jury Presented a modified stability table for complex polynomial [1] which is reduces the computational effort and can also be applied to higher dimensional system. Bistriz et al. mentioned [2] [3] a simplified form for two dimensional stability tests indiscrete time system; it is simplified through the telepolation which gives the benefit of using all the necessary condition as in one dimensional. Bose et al. Demonstrated a stability test [4] based on interlacing (or) separation property, it is to be implemented by using the rational operation only. The stability of two dimensional system can be extended to two dimensional continuous and computed in two methods. First is based on the constrained optimization and second is based on the geometrical constrained of the whole problem, was proven by [5]. Agathoklis et al. suggested [6] that the stability of two dimensional is often required to evaluate the mean square value of quantization error in the output. Myung Ho Pee et al. Proposed two approaches for achieving the stability [7]. The first approach uses PLSI technique and second approach uses Hilbert transform technique. Karan implemented a new procedure for the new stability test where the number of computation [8] is smaller and the result can be extended. The main objective of [9] is to offer a method for checking the stability and compared against Haung. Antoniou et al Focused to reduce the stability criterion of two dimensional systems to stability criterion of one dimensional system [10]. Kanellakis et al. put forwarded a new stability testing algorithm [11] where Xiao yang uses the in term polynomial of two dimensional system. Goodman revealed a detailed discussion [12] of stability in effect of inner polynomial. Zidong et al. made an advent to the problem of robust stability analysis [13] by finding the linear case and investigated within the overflow of non-linearity. Wang, et al collaborated some simple numerical algorithm for two dimensional systems. The simplicity and efficiency of this method will be explained [14] using some interesting numerical examples. Xiao Yang, et al presented a new stability test algorithm for 2D [15] and it used the inner term polynomial of 2D system. Ahmed proposed that the stability [16] which is based on the state space representation which is developed for 2D system. Ooba et al. presented different approach by making use of simple recurrence formula [17] in bilateral quadratic form. Shanks et al. presented the 2D [18] which is conveniently described in terms of 2D Z-transform. Jury proposed a new definition in matrix theory [19]. Woods explained the correspondence stability of 2D filter [20] is proven directly in terms of Z-transform. Lu presented a study on robust stability of 2-D system [21]. Huang discussed about the stability problem of 2-D in [22] and also simplified version should be derived in this concept. Jury et al. had proposed that the stability [23] which is used to check the BIBO stability. It is based on inverse 2-D Laplace transformation. Karivaratha Rajan had presented the paper [24] which is used to test discrete scattering Hurwitz polynomial. The necessary and sufficient condition also is verified. Bisiacco et al. represents the transfer function [25] it was taken as a starting point for 
clarifying the connection between 2-D and BIBO system. Xiheng Hu et al. modified [26] this paper and the polynomial array is developed. This array should be based on linking and further development. Katbab et al. proposed determination of conservative coefficient space with in the coefficient of real 2-D discrete system [27]. Jury et al. described about the application of stability testing [28] to the study of 2-D system and the design of output feedback system. Polyak et al. proposed frequency domain criterion in [29] can be extended in many direction and the problem deals with the three cases in all the three cases the frequency domain approach seems to be promising. Multidimensional linear shift-invariant digital filters are reviewed and extended by Benidirin [30] also the geometrical properties of the stability domain of particular recursive filters were studied. Kurosawa et al. in [31] has presented an efficient algorithm which computes the determinant of a polynomial matrix. A polynomial time stability test algorithm for two-dimensional digital systems was obtained from Anderson method. However, they require huge amount of computations time for all but low order transfer functions.

\section{Proposed Method}

The two dimensional (2-D) linear time invariant discrete system described by its transfer function [22].

$$
G\left(Z_{1}, Z_{2}\right)=\frac{A\left(Z_{1}, Z_{2}\right)}{B\left(Z_{1}, Z_{2}\right)}
$$

where $A\left(z_{1}, Z_{2}\right)$ and $B\left(Z_{1}, Z_{2}\right)$ are coprime polynomials in the independent complex variables $Z_{1}$ and $Z_{2}$. The two dimensional system is stable if and only if $B\left(Z_{1}, Z_{2}\right) \neq 0$ in $\left|Z_{1}\right| \leq 1$ and $\left|Z_{2}\right| \leq 1$. This stability theorem implies that the filter is BIBO stable [18]. Another important stability criterion for 2D linear system was proposed by Huang [22].

$B\left(z_{1}, z_{2}\right) \neq 0$ in $\left|z_{1}\right| \leq 1,\left|z_{2}\right| \leq 1$, if and only if

$$
\text { 1) } B\left(z_{1}, 0\right) \neq 0,\left|z_{1}\right| \leq 1
$$

2) $B\left(z_{1}, z_{2}\right) \neq 0,\left|z_{1}\right| \leq 1,\left|z_{2}\right| \leq 1$

Let $B\left(Z_{1}, Z_{2}\right)=\left(1-Z_{1}\right) Z_{2}+\left(5+Z_{1}\right)=0$

1) With $Z_{2}=0, B\left(Z_{1}\right)=5+Z_{1}=0$, Taking reciprocal of $Z_{1}$, then $\left[\frac{1}{Z_{1}}\right]=X$ (or) $\left[\frac{1}{X}\right]=Z_{1}$

Then $B\left(\frac{1}{Z_{1}}\right)=T_{1}(x)=5 X+1=0$, therfore $|X|=\frac{1}{5}<1$ (Satiesfied).

2) With $Z_{1}=0, B\left(Z_{2}\right)=Z_{2}+5=0$, reciprocal of $Z_{2},\left[\frac{1}{Z_{2}}\right]=X$ (or) $\left[\frac{1}{X}\right]=Z_{2}$

Then $B\left(\frac{1}{Z_{2}}\right)=T_{2}(x)=1+5 X=0$, therefore $|X|=\frac{1}{5}<1$ (Satiesfied).

Condition (2) reduces to a 1-dimenasioanl stability test and is computationally trivial to implement. However, condition (3) is rather difficult to test. Form above example the method proposed by Huang [22] to check the necessary and sufficient conditions involves more arithmetic operations and complexity which can be overcome by the proposed necessary and sufficient conditions.

In general the following 2D characteristics equation [23] can also be chosen:

$$
B\left(Z_{1}, Z_{2}\right)=T_{0}\left(Z_{1}\right) Z_{2}^{n}+T_{1}\left(Z_{1}\right) Z_{2}^{n-1}+\cdots+T_{n}\left(Z_{1}\right)=0
$$

The reciprocals of $Z_{1}$ and $Z_{2}$ are $\left(\frac{1}{Z_{1}}\right)$ and $\left(\frac{1}{Z_{2}}\right)$ respectively are utilized so that the Equation (4) is rewritten as

$$
B\left(\frac{1}{Z_{1}}, \frac{1}{Z_{2}}\right)=T_{0}\left(\frac{1}{Z_{1}}\right)\left(\frac{1}{Z_{2}}\right)^{n}+T_{1}\left(\frac{1}{Z_{1}}\right)\left(\frac{1}{Z_{2}}\right)^{n-1}+\cdots+T_{n}\left(\frac{1}{Z_{1}}\right)=0
$$


Again Equation (5) is represented as

$$
\left.M\left(Z_{1}, Z_{2}\right)\right|_{Z_{1}=Z_{2}=x}=F(Z)=0
$$

$F(x)=0$ is one dimensional equation and for stability $|x|<1$.

$$
\text { Let } F(x)=a_{n} x^{n}+a_{n-1} x^{n-1}+\cdots+a_{0}=0
$$

where $a_{i}$ is the coefficients and $n$ is the degree of $f(z)=0$

The Equation (7) is written as the following Equation (8)

$$
f(x)=X^{n}+\left(\frac{a_{n-1}}{a_{n}}\right) X^{n-1}+\cdots+\left(\frac{a_{0}}{a_{n}}\right)=0
$$

For unity shifted [32] unit circle state that in $f(x), z$ is replaced as $x=x+1$.

The Equation (8) can be written as

$$
f(x+1)=(x+1)^{n}+\left(\frac{a_{n-1}}{a_{n}}\right)(x+1)^{n-1}+\cdots+\left(\frac{a_{0}}{a_{n}}\right)=f(x)
$$

Then the unity shifted $f(x)$ can be analyzed by algebraic method using following necessary and sufficient condition for stability. The proof for the above equations is discussed by Jury in [32].

\section{Proposed Necessary Conditions}

All the roots of $f(x)$ will have negative real parts only and no roots on unit circle [32].

1) $f(0)>0$.

2) $f(-2)>0$ For [Even $f(x)]$.

3) $f(-2)<0$ For [Odd $f(x)]$.

For absolutely stable system, All the roots $f(x)$ must lies between -2 to $0 \quad\left(-2<X_{\text {real }}<0\right)$.

The necessary condition for stability $\left|a_{0}\right|<a_{n}$.

Proposed Sufficient Conditions:

Using the coefficients of $f(x)$ from Equation (9) two triangular matrices is written.

$$
\begin{gathered}
{[X]=\left(\begin{array}{cccccc}
a_{n} & a_{n-1} & a_{n-2} & \cdots & \cdots & a_{0} \\
0 & a_{n} & a_{n-2} & \cdots & \cdots & \cdots \\
0 & 0 & a_{n} & \cdots & \cdots & \cdots \\
0 & 0 & 0 & a_{n} & \cdots & \cdots \\
\cdots & \cdots & \cdots & \cdots & \cdots & \cdots \\
\cdots & \cdots & \cdots & \cdots & \cdots & a_{n}
\end{array}\right)} \\
{[Y]=\left(\begin{array}{ccccc}
a_{n} & a_{n-1} & a_{n-2} & \cdots & a_{0} \\
a_{n-1} & a_{n-2} & a_{n-3} & \cdots & 0 \\
a_{n-2} & a_{n-3} & 0 & \cdots & 0 \\
\vdots & \vdots & \vdots & \ddots & \vdots \\
a_{0} & 0 & 0 & \cdots & 0
\end{array}\right)}
\end{gathered}
$$

From $X$ and $Y$ we construct matrix $H$ [31].

$H=X+Y$

$$
H=\left(\begin{array}{ccccc}
2 a_{n} & 2 a_{n-1} & 2 a_{n-2} & \cdots & 2 a_{0} \\
a_{n-1} & a_{n}+a_{n-2} & a_{n-1}+a_{n-3} & \cdots & \vdots \\
a_{n-2} & a_{n-3} & a_{n} & \cdots & \vdots \\
\vdots & \vdots & \vdots & \ddots & \vdots \\
a_{0} & 0 & 0 & \cdots & a_{n}
\end{array}\right)
$$


A square matrix $H$ is said to be positive inner-wise [33] [34] when all the determinants starting with the centre element(s) and processing outwards up to entire matrix are positive, then the system is asymptotically stable. The proposed necessary and sufficient conditions from the Equations (10) to (14) are applied for the following illustrations. The system is said to be stable only it satisfies all necessary and sufficient conditions.

\section{Illustrations}

EXAMPLE 1: [24]

$$
f(Z)=\left(1-Z_{1}\right) Z_{2}+\left(5+Z_{1}\right)=0
$$

Convert the two dimensional equation into one dimensional equation by taking inverse of variable $Z$.

$$
f(Z)=\frac{1}{Z_{2}}-\frac{1}{Z_{1} Z_{2}}+5+\frac{1}{Z_{1}}=0
$$

Put $Z_{1}=Z_{2}=Z$

$$
\begin{gathered}
f(Z)=\frac{1}{Z}-\frac{1}{Z^{2}}+5+\frac{1}{Z}=0 \\
f(Z)=5 Z^{2}+2 Z-1=0
\end{gathered}
$$

For unity shifting the unit circle to left half of Z-plane $(X=Z-1)$ put $Z=X+1$

$$
\begin{gathered}
f(X+1)=5(X+1)^{2}+2(X+1)-1=0 \\
f(X+1)=f(X)=5 X^{2}+12 X+6=0 \\
f(x)=5 X^{2}+12 X+6=0
\end{gathered}
$$

Test for Necessary Conditions:

$$
\begin{gathered}
f(0)=6>0(\text { satisfied }) \\
f(-2)=2>0(\text { satisfied })
\end{gathered}
$$

Test for Sufficient Conditions:

To check the requirements of sufficiency, we construct the matrix $X$ and $Y$.

$$
X=\left(\begin{array}{ccc}
5 & 12 & 6 \\
0 & 5 & 12 \\
0 & 0 & 5
\end{array}\right) \quad Y=\left(\begin{array}{ccc}
5 & 12 & 6 \\
12 & 6 & 0 \\
5 & 0 & 0
\end{array}\right)
$$

Add the matrix $X$ and $Y$.

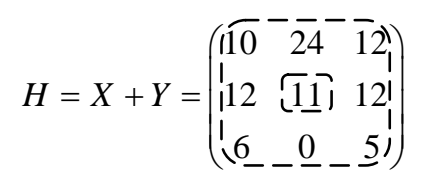

The determinants are

$$
\begin{gathered}
\nabla_{1}=11>0(\text { satisfied }) \\
\nabla_{3}=\left|\begin{array}{ccc}
10 & 24 & 12 \\
12 & 11 & 12 \\
6 & 0 & 5
\end{array}\right|=46>0 \text { (Satisfied) }
\end{gathered}
$$

This system satisfies the both necessary conditions and sufficient conditions therefore the given system is absolutely stable.

EXAMPLE 2: [14] 


$$
f(Z)=12+10 Z_{1}+6 Z_{2}+5 Z_{1} Z_{2}+2 Z_{1}^{2}+Z_{1}^{2} Z_{2}=0
$$

Convert the two dimensional equation into one dimensional equation by taking inverse of variable $Z$.

$$
f(Z)=12+\frac{10}{Z_{1}}+\frac{6}{Z_{2}}+\frac{5}{Z_{1} Z_{2}}+\frac{2}{Z_{1}^{2}}+\frac{1}{Z_{1}^{2} Z_{2}}=0
$$

Put $Z_{1}=Z_{2}=Z$

$$
\begin{gathered}
f(Z)=12+\frac{10}{Z}+\frac{6}{Z}+\frac{5}{Z^{2}}+\frac{2}{Z^{2}}+\frac{1}{Z^{3}}=0 \\
f(Z)=12 Z^{3}+16 Z^{2}+7 Z+1=0
\end{gathered}
$$

For unity shifting the unit circle put $Z=X+1$

$$
\begin{gathered}
f(X+1)=12(X+1)^{3}+16(X+1)^{2}+7(X+1)+1=0 \\
f(X+1)=f(X)=12 X^{3}+52 X^{2}+75 X+36=0
\end{gathered}
$$

Test for Necessary Conditions:

$$
\begin{gathered}
f(0)=36>0(\text { satisfied }) \\
f(-2)=-2>0(\text { satisfied })
\end{gathered}
$$

Test for Sufficiency Conditions:

To check the requirements of sufficiency, we construct $X$ and $Y$ :

$$
X=\left(\begin{array}{cccc}
12 & 52 & 75 & 36 \\
0 & 12 & 52 & 75 \\
0 & 0 & 12 & 52 \\
0 & 0 & 0 & 12
\end{array}\right) Y=\left(\begin{array}{cccc}
12 & 52 & 75 & 36 \\
52 & 75 & 36 & 0 \\
75 & 36 & 0 & 0 \\
36 & 0 & 0 & 0
\end{array}\right)
$$

Add the matrix $X$ and $Y$

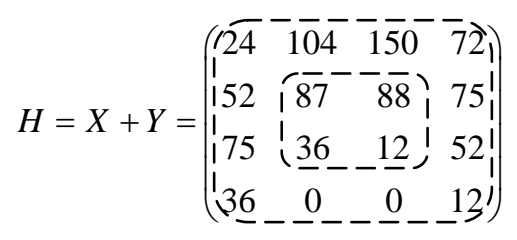

The determinants are

$$
\begin{gathered}
\nabla_{2}=\left|\begin{array}{ll}
87 & 88 \\
36 & 12
\end{array}\right|=-2124<0(\text { Not satisfied }) \\
\nabla_{4}=\left|\begin{array}{cccc}
24 & 104 & 150 & 72 \\
52 & 87 & 88 & 75 \\
75 & 36 & 12 & 52 \\
36 & 0 & 0 & 12
\end{array}\right|=-34120<0 \text { (Not satified) }
\end{gathered}
$$

This system satisfies the both necessary conditions but doesn't satisfy sufficient conditions therefore the given system is unstable.

EXAMPLE 3: [31]

$$
f(Z)=\left(2.4+1.2 Z_{2}\right)+\left(3.2+1.6 Z_{2}\right) Z_{1}+\left(1.4+0.7 Z_{2}\right) Z_{1}^{2}+\left(0.2+0.1 Z_{2}\right) Z_{1}^{3}=0
$$

Convert the two dimensional equation into one dimensional equation by taking inverse of variable-Z.

$$
f(Z)=2.4+\frac{1.2}{Z_{2}}+\frac{3.2}{Z_{1}}+\frac{1.6}{Z_{1} Z_{2}}+\frac{1.4}{Z_{1}^{2}}+\frac{0.7}{Z_{1}^{2} Z_{2}}+\frac{0.2}{Z_{1}^{3}}+\frac{0.1}{Z_{2} Z_{1}^{3}}=0
$$


Put $Z_{1}=Z_{2}=Z$

$$
\begin{gathered}
f(Z)=2.4+\frac{1.2}{Z}+\frac{3.2}{Z}+\frac{1.6}{Z^{2}}+\frac{1.4}{Z^{2}}+\frac{0.7}{Z^{3}}+\frac{0.2}{Z^{3}}+\frac{0.1}{Z^{4}}=0 \\
f(Z)=2.4 Z^{4}+4.4 Z^{3}+3 Z^{2}+0.9 Z+0.1=0
\end{gathered}
$$

For unity shifting the unit circle put $Z=X+1$

$$
\begin{gathered}
f(X+1)=2.4(X+1)^{4}+4.4(X+1)^{3}+3(X+1)^{2}+0.9(X+1)+0.1=0 \\
f(X+1)=f(X)=2.4 X^{4}+14 X^{3}+30.6 X^{2}+29.7 X+10.8=0
\end{gathered}
$$

Test for Necessary Conditions:

$$
\begin{aligned}
& f(0)=10.8>0(\text { satisfied }) \\
& f(-2)=0.2>0(\text { satisfied })
\end{aligned}
$$

Test for Sufficient Conditions:

To check the requirements of sufficiency, we construct $X$ and $Y$ :

$$
X=\left(\begin{array}{ccccc}
2.4 & 14 & 30.6 & 29.7 & 10.8 \\
0 & 2.4 & 14 & 30.6 & 29.7 \\
0 & 0 & 2.4 & 14 & 30.6 \\
0 & 0 & 0 & 2.4 & 14 \\
0 & 0 & 0 & 0 & 2.4
\end{array}\right) \quad Y=\left(\begin{array}{ccccc}
2.4 & 14 & 30.6 & 29.7 & 10.8 \\
14 & 30.6 & 29.7 & 10.8 & 0 \\
30.6 & 29.7 & 10.8 & 0 & 0 \\
29.7 & 10.8 & 0 & 0 & 0 \\
10.8 & 0 & 0 & 0 & 0
\end{array}\right)
$$

Add the matrix $X$ and $Y$

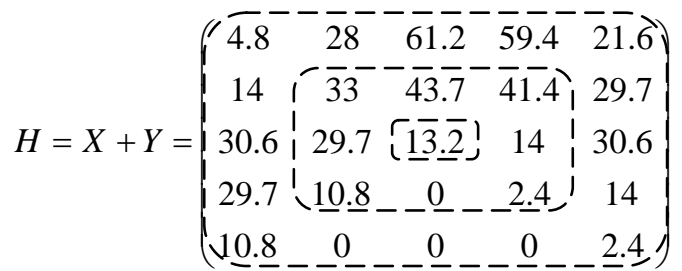

The determinants are

$$
\begin{gathered}
\nabla_{1}=13.2>0(\text { satisfied }) \\
\nabla_{3}=\left|\begin{array}{ccc}
33 & 43.7 & 41.4 \\
29.7 & 13.2 & 14 \\
10.8 & 0 & 2.4
\end{array}\right|=-1364.04<0(\text { Not satified }) \\
\nabla_{5}=\left|\begin{array}{ccccc}
4.8 & 28 & 61.2 & 59.4 & 21.6 \\
14 & 33 & 43.7 & 41.4 & 29.7 \\
30.6 & 29.7 & 13.2 & 14 & 30.6 \\
29.7 & 10.8 & 0 & 2.4 & 14 \\
10.8 & 0 & 0 & 0 & 2.4
\end{array}\right|=-674.98<0 \text { (Not satisfied) }
\end{gathered}
$$

This system satisfies the necessary conditions and doesn't satisfy sufficient conditions therefore the given system is unstable.

\section{Result and Discussion}

Three examples were presented in this paper to check the stability of the given two-dimensional characteristics polynomial. It has to be noted that the method proposed by Jury in 1971 [19] to check the stability of the given 
characteristics polynomial necessary conditions and sufficient conditions must be satisfied. In sufficient condition two matrices were formed like $H=X+Y$ and $H=X-Y$ then the inner determinants were determined, but in the proposed method necessary condition is checked as per Jury method and for checking the sufficient conditions only $H=X+Y$ is considered to find the inner determinants, which reduces the number computations and one more important modification has compared with Jury method that accounts all the coefficients of the characteristics equation in order to form the matrix followed by applying left shifting and right shifting principle to form $X$ and $Y$ matrix respectively. The coefficients of this characteristic equation are suitably arranged in the form of $(n+1) \times(n+1)$ matrix and the inner determinants are evaluated using Jury concept. The predominant merit in the proposed method is its versatility in direct application which becomes very simple.

\section{Conclusion}

The implemented procedure for a modified stability test for two-dimensional linear time invariant discrete system is direct and much simpler than the Jury's method. The proposed method had solved the problem in a very low count of arithmetic operations compared to Jury [19] inners approach method.

\section{References}

[1] Jury, E.I. (1988) Modified Stability Table for 2-D Digital Filters. IEEE Transactions on Circuits \& Systems, 35, 116-119. http://dx.doi.org/10.1109/31.1707

[2] Bristriz, Y. (2001) Stability Testing of 2-D Discrete Linear Systems by Telepolation of an Immittance-Type Tabular Test. IEEE Transactions on Circuits and Systems I: Fundamental Theory and Applications, 48, 840-846. http://dx.doi.org/10.1109/81.933325

[3] Bristriz, Y. (2004) Testing Stability of 2-D Discrete Systems by a Set of Real 1-D Stability Tests. IEEE Transactions on Circuits and Systems I: Regular Papers, 51, 1312-1320. http://dx.doi.org/10.1109/TCSI.2004.830679

[4] Bose, N.K and Jury, E.I. (1975) Inner Algorithm to Test for Positive Definiteness of Arbitrary Binary Forms. IEEE Transaction on Automatic Control, 20, 169-170.

[5] Mastorakis, N.E. (1998) A Method for Computing the 2-DStability Margin. IEEE Transactions on Circuits \& Systems-II, Analog \& Digital Signal Processing, 45, 376-378. http://dx.doi.org/10.1109/82.664243

[6] Agathoklis, P., Jury, E.I. and Mansour, M. (1993) Algebraic Necessary \& Sufficient Conditions for the Stability of 2-D Discrete Systems. IEEE Transactions on Circuits \& Systems-II, Analog \& Digital Signal Processing, 40, 257-258. http://dx.doi.org/10.1109/82.224316

[7] Pee, M., Khargonekar, P. and Lee, E. (1986) Further Result on Possible Root Locations of 2-D Polynomial. IEEE Transactions on Circuits \& Systems, 33, 566-569. http://dx.doi.org/10.1109/TCS.1986.1085944

[8] Karan, B.M. and Srivastava, M.C. (1986) A New Stability Test for Two-dimensional Filters. IEEE Transactions on Circuits \& Systems, 33, 807-809. http://dx.doi.org/10.1109/TCS.1986.1085999

[9] Anderson, B.D.O. and Jury, E.L. (1973) Stability Test for Two-Dimensional Recursive Filters. IEEE Transactions on Audio and Electroacoustics, 20, 158-163.

[10] Antoniou, G.E., Vardoulakis, S.J. and Paraskevopoulos, P.N. (1990) A Simple Stability Test for 2-D Systems. IEEE Transactions on Circuits \& Systems, 37, 972-974. http://dx.doi.org/10.1109/31.55078

[11] Kanellakis, A., Theodorou, N. and Tzafestas, S. (1989) A New Stability Test for Two-dimensional Discrete Systems using Bilinear Continuous Fractions. IEEE Transactions on Circuits \& Systems, 36, 141-143. http://dx.doi.org/10.1109/31.16580

[12] Goodman, D. (1977) Some Stability Properties of Two-Dimensional Linear Shift-Invariant Digital Filters. IEEE Transactions on Circuits \&Systems, 24, 201-208. http://dx.doi.org/10.1109/TCS.1977.1084322

[13] Wang, Z.D. and Liu, X.H. (2003) Robust Stability of Two-Dimensional Uncertain Discrete Systems. IEEE Transactions on Signal Processing, 10, 133-136.

[14] Wang, M., Lee, E.B. and Boley, D. (1992) A Simple Method to Determine the Stability and Margin of Stability of 2-D Recursive Filters. IEEE Transactions on Circuits \& Systems, 39, 237-239. http://dx.doi.org/10.1109/81.128017

[15] Yang, X. and Unbehauen, R. (1998) New Stability Test Algorithm for 2-Dimensional Digital Filters. IEEE Transactions on Circuits \& Systems, 45, 739-741.

[16] Ahmed, A. (1980) On the Stability of Two-Dimensional Discrete Systems. IEEE Transactions on Automatic Control, 25, 551-552. http://dx.doi.org/10.1109/TAC.1980.1102352

[17] Ooba, T. and Funahashi, Y. (2004) Stability Analysis of two-Dimensional Systems by Means of Finitely Constructed 
Bilateral Quadratic Forms. IEEE Transactions on Automatic Control, 49, 2068-2073.

[18] Shanks, J.L. and Justice, J.H. (1972) Stability and Synthesis of Two-Dimensional Recursive Filters. IEEE Transactions on Audio and Electroacoustic, 20, 115-128. http://dx.doi.org/10.1109/TAU.1972.1162358

[19] Jury, E.I. (1971) Inners-Approach to Some Problems of System Theory. IEEE Transactions on Automatic Control, 16, 233-240. http://dx.doi.org/10.1109/TAC.1971.1099725

[20] Woods, J.W. (1983) Stability of Two-Dimensional Causal Digital Filters Using the Residue Theorem. IEEE Transactions on Acoustics, Speech \& Signal Processing, 31, 774-775. http://dx.doi.org/10.1109/TASSP.1983.1164106

[21] Lu, W.S. (1995) On Robust Stability of 2-D Discrete Systems. IEEE Transactions on Automatic Control, 40, 502-506. http://dx.doi.org/10.1109/9.376069

[22] Huang, T.S. (1972) Stability of Two-Dimensional Recursive Filters. IEEE Transactions on Audio and Electroacoustic, 20, 158-163. http://dx.doi.org/10.1109/TAU.1972.1162364

[23] Jury, E.I. and Bauer, P. (1988) On the Stability of Two-Dimensional Continuous Systems. IEEE Transactions on Circuits and Systems, 35, 1487-1500. http://dx.doi.org/10.1109/31.9912

[24] Karivaratha Rajan, P. and Reddy, H.C. (1989) A Test Procedure for 2-D Discrete Scattering Hurwitz Polynomials. IEEE Transactions on Acoustics, Speech and Signal Processing, 37, 118-120. http://dx.doi.org/10.1109/29.17508

[25] Bisiacco, M.E. and Marchesini, G. (1985) On Some Connections between BIBO \& Internal Stability of Two-Dimensional Filters. IEEE Transactions on Circuits and Systems, 32, 948-953. http://dx.doi.org/10.1109/TCS.1985.1085801

[26] Hu. X.H. and Jury, E.I. (1995) On 2-Dimensional Filter Stability Test. IEEE Transactions on Circuits \& Systems, 41, 457-462.

[27] Katbab, A. and Jury, E.I. (1992) Robust Stability of Two-Dimensional Digital Filters under Coefficient Perturbation. IEEE Transactions on signal processing, 40, 993-996. http://dx.doi.org/10.1109/78.127976

[28] Jury, E.I. and Gutman, S. (1975) On the Stability of the A Matrix inside the Unit Circle. IEEE Transaction on Automatic Control, 20, 533-535.

[29] Polyak, B.T. and Shmulyian, S.B. (1994) Frequency Domain Criteria for Robust Stability of Bivariate Polynomial. IEEE Transactions on Circuits \& Systems, 41, 161-167. http://dx.doi.org/10.1109/81.269052

[30] Benidir, M. (1991) Sufficient Conditions for the Stability of Multidimensional Recursive Digital Filters. Proceedings of the International Conference on Acoustics, Speech and Signal Processing, Vol. 4, 2885-2888. http://dx.doi.org/10.1109/icassp.1991.151005

[31] Kurosawa, K., Yamada, I., Yokokawa, T. and Tsujii, S. (1993) A Fast Stability Test for Multidimensional Systems. IEEE International Symposium on Circuits and Systems, Tokyo Institute of Technology, Vol. 152, 579-582.

[32] Jury, E.I. (1976) Inners and Stability of Dynamic Systems. Wiley Inter Science, New York, 42-43.

[33] Nagrath, I.J. and Gopal, M. (1996) Control Systems Engineering. New Age International (p) Limited, New Delhi, 424-429.

[34] Jury, E.I. (1964) Theory and Application of the Z-Transform Method. John Wiley and Sons, Inc., New York. 